\title{
Reynolds stress tensor and pressure-related turbulence transport terms measured by time-resolved tomographic-PIV
}

\author{
J.R. Moreto ${ }^{1}$, X. Liu ${ }^{1 *}$ \\ ${ }^{1}$ San Diego State University, Department of Aerospace Engineering, San Diego, California, USA \\ *Xiaofeng.Liu@sdsu.edu
}

\begin{abstract}
Turbulence is inherently a three-dimensional and time dependent flow phenomenon (Pope, 2001). Because of the ubiquitous existence of turbulent flows in nature, accurate characterization of turbulent flows, either through experimental measurements or through direct numerical simulations, is of paramount importance for modeling turbulence (Liu and Katz, 2018). Since its inception in 1984 (Adrian, 1984), Particle Image Velocimetry (PIV), among several other conventional techniques used for turbulence measurements, has been a valuable tool for providing reliable experimental data for turbulence research. Several advancements in hardware such as high-speed cameras, together with innovative algorithms and procedures, have extended the scope of PIV to a variety of applications. Westerweel et al. (2013) point out in a recent review article that one of the main advantages of the PIV measurement is its unique ability in measuring quantitatively spatial derivatives of the flow field. With the development of Tomographic PIV introduced by Elsinga et al. (2006), it is now possible to measure simultaneously the distributions of three velocity components in a threedimensional flow field, thus enabling us to measure all the velocity derivatives of a turbulent flow. However, for a thorough characterization of a turbulent flow, in addition to the velocity gradients, the instantaneous pressure distribution in the 3D flow field also needs to be measured.

The instantaneous pressure distribution in a turbulent flow field can be measured non-intrusively by integrating the measured material acceleration using particle image velocimetry (PIV), as demonstrated by Liu and Katz (2006, 2008, 2013), Joshi et al. (2014), Van Oudheusden (2008) and Ragni et al. (2009), to name a few. The pressure can also be obtained by solving the Poisson equation, as shown in Violato et al. (2011), and De Kat and Van Oudheusden (2012). Review and comparison of the two pressure reconstruction approaches can be found in Charonko et al. (2010) and Van Oudheusden (2013). Liu and Moreto (2020) demonstrate the robustness and low noise sensitivity of the rotating parallel ray omnidirectional integration method, which is capable of measuring the instantaneous pressure distributions at high accuracy in a complex turbulent flow field. Coupled with the time-resolved Tomographic PIV, the pressure reconstruction method enables the experimental characterization of all the terms including the pressure-related turbulence transport terms in the Reynolds Stress Transport equation.

Liu and Katz (2018) applied planar PIV in conjunction with the virtual boundary omni-directional integration to the study of a shear layer flow impinging on a cavity trailing corner at a Reynolds number of $4 \times 10^{4}$. They found that the distribution patterns of the pressure diffusion and the turbulence diffusion differ considerably, indicating that the conventional modeling for the transport terms is not adequate, at least for the turbulent shear layer flow over a cavity. Their results also show that the turbulence fluctuation energy is redistributed from the streamwise component to the lateral ones, and this intercomponent energy transfer has an important impact on the flow dynamics around the cavity trailing corner area. However, due to the limitation in planar PIV, they can only infer indirectly the spanwise intercomponent turbulence energy transfer based on the measured streamwise and wall normal components of the pressure-rate-of strain terms. In this paper, we will demonstrate the capability of measuring simultaneously all the Reynolds stress tensor components and the instantaneous three dimensional distribution of pressure for a cavity flow at a Reynolds number of $4 \times 10^{4}$ by Time-Resolved Tomographic PIV. The free stream velocity is set to $1.2 \mathrm{~m} / \mathrm{s}$, which is identical to the Hopkins experiment (Liu and Katz, 2013, 2018). The 2D cavity geometry is $38.1 \mathrm{~mm}$ long, $101.6 \mathrm{~mm}$ wide and $30.0 \mathrm{~mm}$ deep, with the beginning part of the upstream ramp machined with tripping grooves. Except the width, the geometry of cavity is also identical to that of the Hopkins cavity setup, thus facilitating comparison and validation of the new Tomo-PIV measurement results. Preliminary results on
\end{abstract}


the Reynolds normal stress measurement around the cavity trailing corner based on a limited sample of 149 instantaneous realizations (thus not converged yet) are shown in Figure 1. Time-averaged pressure distribution around the cavity trailing corner based on 4076 realizations (also not converged yet) is shown in Figure 2. The selected image acquisition rate $(4996 \mathrm{~Hz})$ is sufficient to resolve the Kolmogorov time scale based on a curve fit to the spatial energy spectra (Liu and Katz, 2013, 2018), according to which, the Kolmogorov length scale is $26 \mu \mathrm{m}$ and the Taylor transverse microscale is $0.5 \mathrm{~mm}$. The size of the Tomo-PIV measurement volume in the current study is approximately $42.6 \times 11.7 \times 6.9 \mathrm{~mm}^{3}$ to maintain sufficient resolution. In the current experiment, an interrogation volume of 40 pixel $\times 40$ pixel $\times 40$ pixel, which corresponds to $0.84 \mathrm{~mm} \times 0.84 \mathrm{~mm} \times 0.84 \mathrm{~mm}$ in physical dimension, is compatible with the Taylor transverse microscale, but one order of magnitude larger than the Kolmogorov length scale. A 75\% overlap between the interrogation windows gives a vector spacing of $0.21 \mathrm{~mm}$. In this paper, based on 140,000 instantaneous 3D realizations of the cavity flow, we will present converged turbulence statistics on all terms in the Reynolds stress transport equation, with an emphasis on the characterization of the magnitude of the intercomponent turbulence energy fluctuations represented by the pressure-rate-of strain terms, so as to verify the conjecture raised in Liu and Katz (2018) about the magnitude of the third component of the intercomponent energy transfer.

a)
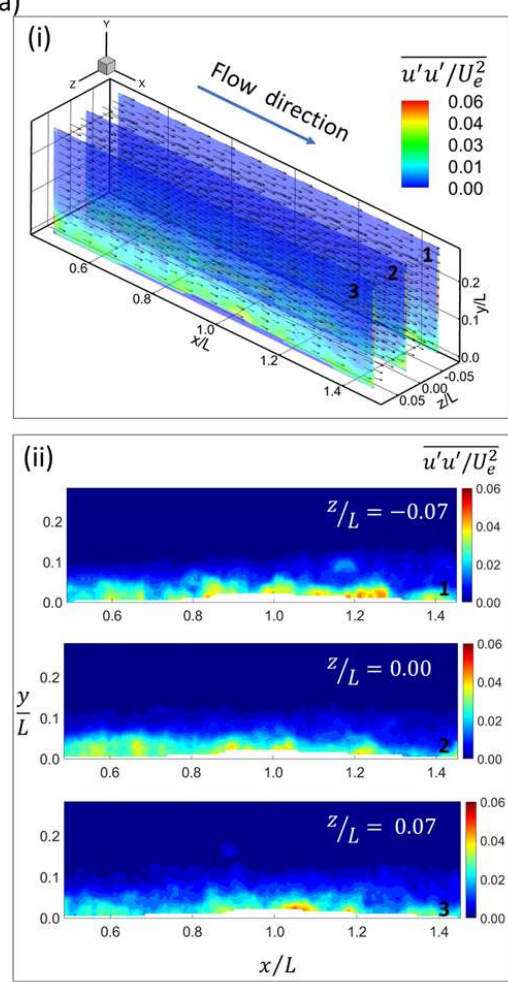

b)
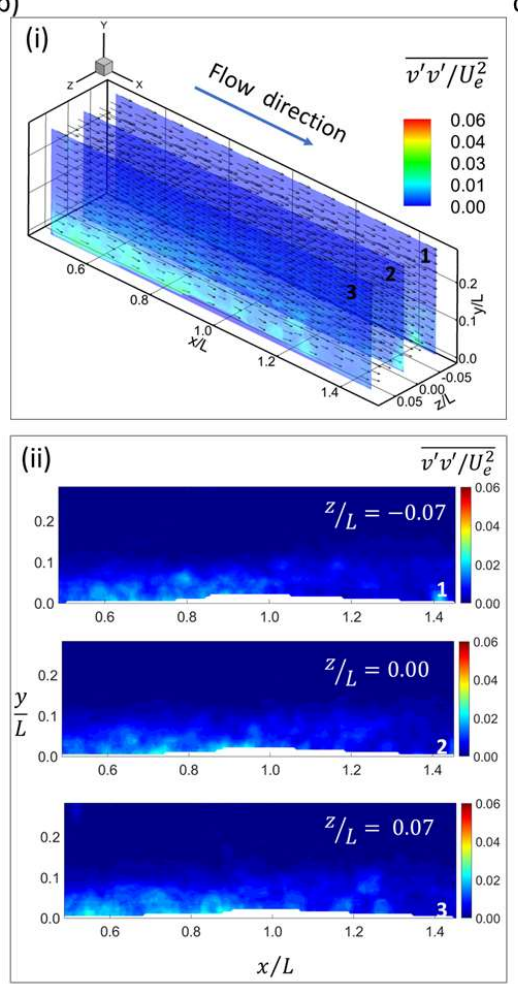

c)
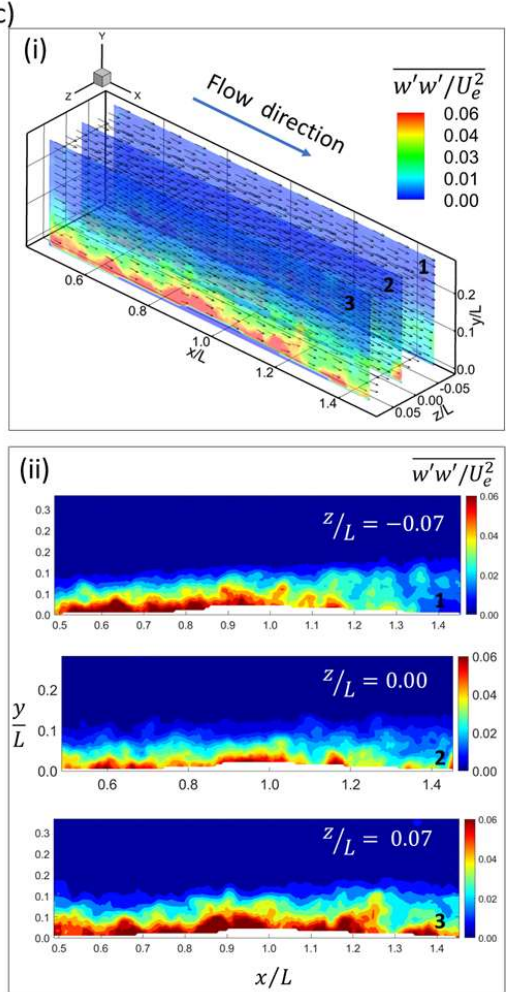

Figure 1: Normal Reynolds stress profiles based on 149 realizations of Tomo-PIV measurement over a cavity trailing corner at a Reynolds number of $4 \times 10^{4}$ (a) $\overline{u^{\prime} u^{\prime}}$, (b) $\overline{v^{\prime} v^{\prime}}$, (c) $\overline{w^{\prime} w^{\prime}}$ distributions, which consist of, (i) 3D distributions, and (ii) planar contours at three selected spanwise planes that are located at the center of the measurement volume, and at the places close to the two edges of measurement volume in the spanwise direction, respectively.

\section{Acknowledgements}

This work has been sponsored by the Office of Naval Research Grant No. N00014-20-1-2276 (Dr. Ki-Han Kim is the Program Officer). Optics on loan from the Naval Surface Warfare Center Carderock Division is gratefully acknowledged. 


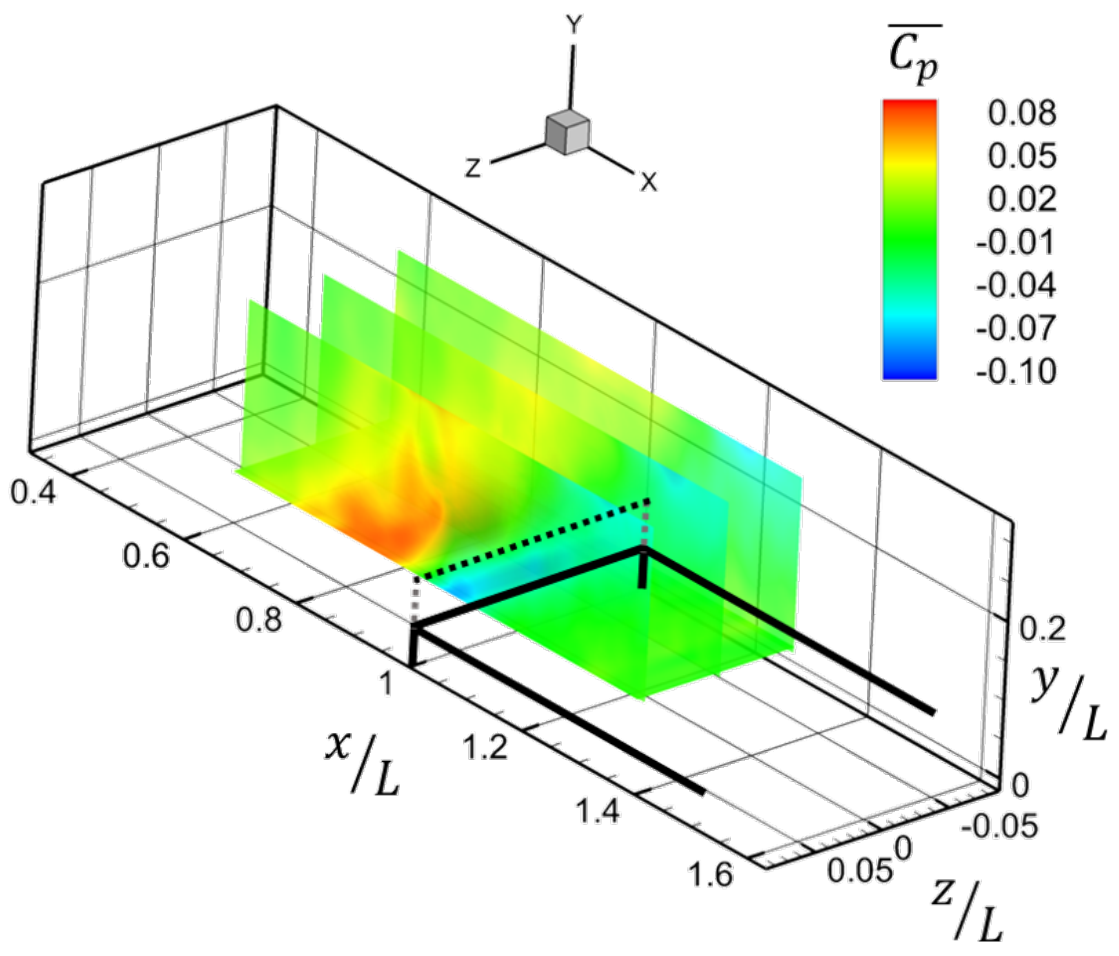

Figure 2: Time-averaged pressure distribution around the cavity trailing corner based on 4076 realizations.

\section{References}

Adrian RJ (1984) Scattering particle characteristics and their effect on pulsed laser measurements of fluid flow: speckle velocimetry vs particle image velocimetry. Applied optics 23:1690-1691

Charonko JJ, King CV, Smith BL, and Vlachos PP (2010) Assessment of pressure field calculations from particle image velocimetry measurements. Measurement Science and technology 21:105401

De Kat R and Van Oudheusden B (2012) Instantaneous planar pressure determination from piv in turbulent flow. Experiments in fluids 52:1089-1106

Elsinga GE, Scarano F, Wieneke B, and van Oudheusden BW (2006) Tomographic particle image velocimetry. Experiments in fluids 41:933-947

Joshi P, Liu X, and Katz J (2014) Effect of mean and fluctuating pressure gradients on boundary layer turbulence. Journal of fluid mechanics 748:36-84

Liu X and Katz J (2006) Instantaneous pressure and material acceleration measurements using a fourexposure piv system. Experiments in fluids 41:227-240

Liu X and Katz J (2008) Cavitation phenomena occurring due to interaction of shear layer vortices with the trailing corner of a two-dimensional open cavity. Physics of fluids 20:041702

Liu X and Katz J (2013) Vortex-corner interactions in a cavity shear layer elucidated by time-resolved measurements of the pressure field. Journal of Fluid Mechanics 728:417-457 
Liu X and Katz J (2018) Pressure-rate-of-strain, pressure diffusion, and velocity-pressure-gradient tensor measurements in a cavity flow. AIAA Journal 56:3897-3914

Liu X and Moreto JR (2020) Error propagation from the piv-based pressure gradient to the integrated pressure by the omnidirectional integration method. Measurement Science and Technology 31:055301

Pope SB (2001) Turbulent flows

Ragni D, Ashok A, Van Oudheusden B, and Scarano F (2009) Surface pressure and aerodynamic loads determination of a transonic airfoil based on particle image velocimetry. Measurement Science and technology 20:074005

Van Oudheusden B (2008) Principles and application of velocimetry-based planar pressure imaging in compressible flows with shocks. Experiments in fluids 45:657-674

Van Oudheusden B (2013) Piv-based pressure measurement. Measurement Science and Technology 24:032001

Violato D, Moore P, and Scarano F (2011) Lagrangian and eulerian pressure field evaluation of rod-airfoil flow from time-resolved tomographic piv. Experiments in fluids 50:1057-1070

Westerweel J, Elsinga GE, and Adrian RJ (2013) Particle image velocimetry for complex and turbulent flows. Annual Review of Fluid Mechanics 45:409-436 